\title{
Linx
}

Revue des linguistes de l'université Paris X Nanterre

46 | 2002

Les connecteurs

\section{Construction des énoncés et connecteurs dans la structuration des récits enfantins en arabe tunisien et en français}

Lamia Allal, Chiraz Anane, Monique Sénémaud et Colette Noyau

\section{OpenEdition}

\section{Journals}

Édition électronique

URL : http://journals.openedition.org/linx/109

DOI : 10.4000/linx.109

ISSN : 2118-9692

Éditeur

Presses universitaires de Paris Nanterre

\section{Édition imprimée}

Date de publication : 1 juin 2002

Pagination : 133-151

ISSN : 0246-8743

\section{Référence électronique}

Lamia Allal, Chiraz Anane, Monique Sénémaud et Colette Noyau, «Construction des énoncés et connecteurs dans la structuration des récits enfantins en arabe tunisien et en français », Linx [En ligne], 46 | 2002, mis en ligne le 25 janvier 2011, consulté le 02 mai 2019. URL : http://

journals.openedition.org/linx/109; DOI : 10.4000/linx.109 


\title{
Construction des énoncés et connecteurs dans la structuration des récits enfantins en arabe tunisien et en français
}

\author{
Lamia ALLAL*, Chiraz ANANE**, \\ Monique SÉNÉMAUD*, Colette NOYAU* \\ * UMR 7114 MODYCO CNRS \& Université Paris-X \\ ** Université de Tunis-El Manar
}

Ce travail associe l'analyse de productions narratives d'enfants de cinq à dix ans, en français par des monolingues (Sénémaud, 1997, 1998, thèse en cours ; Choi \& Sénémaud 2000), en arabe tunisien et en français par des enfants tunisiens vivant en Tunisie (Anane, 2001, thèse en cours) et par des enfants issus de l'immigration en France (Allal, 2000, thèse en cours), pour y examiner l'utilisation des connecteurs en tant qu'indice de l'acquisition dans ces différentes situations (langue première unique, langues premières parallèles de statuts différents, langue première et langue étrangère introduite par l'école). Il est comparatif par nature, les études monographiques mises en regard partageant cadre théorique, méthodes de recueil et d'analyses des données (cf. Noyau 1998, Noyau \& Paprocka 2000, Noyau et al. sous presse), et les productions des divers groupes d'enfants étant comparées à celles de locuteurs adultes dans les deux langues de référence.

Nous travaillons de façon privilégiée sur l'acquisition du domaine de la temporalité, du placement dans le temps des procès (actions, événements, activités, états...) dans le récit. Et nous examinons comment l'enfant, l'apprenant, le locuteur, conceptualise des structures événementielles complexes, et les formule en un texte global, dans une situation déterminée de production de récit, compte tenu de son développement cognitif (âges cognitifs des enfants) et du répertoire linguistique dont il dispose. La représentation de structures événementielles par les locuteurs peut être caractérisée par la condensation de linformation (condensation relative des propositions en énoncés complexes intégrant plusieurs propositions dans un même énoncé). C'est pourquoi les résultats de notre étude sont analysés d'abord selon les axes monopropositionnel/pluripropositionnel, puis paratactique /hypotactique.

Dans cette problématique, les connecteurs sont des outils permettant de relier des prédications de procès entre elles et en une structure globale. Pour le récit, il s'agit essentiellement de connecteurs additifs, temporels, causaux, de but, et à titre subsidiaire de connecteurs qui nuancent les relations ci-dessus (par exemple en ajoutant une valeur argumentative : 'mais' pour le contraste entre attentes de polarité positive et attentes de polarité négative dans une relation de consécutivité 
ou de concomitance entre événements), ou des spécifications diverses. Nous postulons que la capacité à construire des énoncés pluripropositionnels est le corrélat de la capacité - conceptuelle - à concevoir différents procès comme liés en un ensemble faisant l'objet d'un seul acte d'énonciation. Nous nous intéressons donc ici au développement de la condensation à travers sa manifestation linguistique, c'est-à-dire à la capacité à rassembler $n$ prédications de procès en des énoncés complexes, dans leurs récits. Pour ce faire, nous adoptons un point de vue fonctionnaliste articulant conceptualisation et formulation (cf. Levelt 1989, Klein \& Stutterheim 1989), et notre travail a pour thème général la condensation (cf. Noyau \& Paprocka 2000, Noyau \& al. sous presse). Nous visons à répondre ici à trois questions :

a) Comment les représentations de procès constituant la micro-structure des textes narratifs produits se trouvent-elles intégrées en des unités énonciatives complexes pluripropositionnelles, au sein desquelles les prédications de procès sont reliées par des connexions des différents types sémantiques évoqués?

b) Peut-on caractériser les productions des différents locuteurs comme ayant un degré élevé ou faible de condensation? On dira que les énoncés monopropositionnels ont un degré 0 de condensation, que les énoncés pluripropositionnels où chacune des prédications garde son indépendance énonciative et sa plénitude de marquage (énoncés paratactiques à propositions juxtaposées ou coordonnées) manifestent un degré de condensation faible, et que les énoncés pluripropositionnels, où certaines des propositions sont mises en dépendance d'autres, avec réduction partielle de leur autonomie et de leur plénitude de marquage (énoncés hypotactiques avec subordination, intégration plus forte par infinitivisation, gérondivisation, nominalisation de prédication entière avec ses arguments) ont un degré de condensation plus élevé.

c) Dans quelle mesure ces relations sont-elles marquées de façon explicite au moyen d'un répertoire plus ou moins approprié et plus ou moins riche de connecteurs, ou bien leur lien est-il implicite, éventuellement signalé par la prosodie, à charge au destinataire de reconstruire le type de relation qu'entretiennent les propositions?

Il s'agit, plus généralement, de caractériser le façonnage, selon les termes de Haiman \& Thompson (1989, 'information packaging', cf. Blanche-Benveniste 1997) de ces représentations propositionnelles, en énoncés dont la structure relationnelle organise le texte.

Nous visons à montrer, à partir des productions textuelles orales des enfants, dans l'acquisition de la langue première (L1), d'une langue étrangère (L2), ou dans la langue dominante vs la langue faible simultanément lors de l'acquisition d'un bilinguisme asymétrique, comment la cohésion et la connexité se développent dans l'acquisition de la langue, notamment par le développement des énoncés complexes, et comment le facteur typologique influe sur ce développement, la langue française et la langue arabe manifestant des tendances divergentes de ce point de vue.

Nous nous appuyons sur des données orales de récits enregistrés puis transcrits, à partir d'une tâche verbale proposée à tous les groupes d'enfants et aux adultes des deux langues, celle de raconter une histoire à partir d'un livre d'images sans texte, 'Frog, where are you ?' (Mayer, 1969), en arabe tunisien et en français ${ }^{1}$.

Les récits ont été segmentés en propositions (clauses) et en énoncés (mono- et pluripropositionnels) pour nos visées, selon des principes développés dans Berman \& Slobin (1995),

\footnotetext{
${ }^{1}$ Ce support a été proposé par Berman \& Slobin (1995) pour une vaste étude translinguistique sur le développement de la capacité narrative chez les enfants. Il sert depuis à de nombreuses recherches psycholinguistiques.
} 
Noyau \& al. (sous presse) et Sanz (1999)2 et qui tiennent compte d'un faisceau d'indices fonctionnels, prosodiques et interprétatifs, les paramètres formels étant un aboutissement, non un point de départ dans des données acquisitionnelles. Ils constituent neuf corpus dont le découpage en propositions est présenté dans le tableau ci-dessous.

Tableau 1 : Nombre des propositions dans les corpus étudiés

\begin{tabular}{|c|c|c|c|c|}
\hline Age & Natifs-LF & $\begin{array}{c}\text { Bilingues- LF (scolarisés } \\
\text { en France) }\end{array}$ & $\begin{array}{c}\text { Bilingues - LA } \\
\text { (scolarisés en France) }\end{array}$ & $\begin{array}{c}\text { Arabophones - } \\
\text { (résidant en Tunisie) } \\
\text { Apprenants -LF }\end{array}$ \\
\hline 7 ans & $\begin{array}{c}5 \text { enfants } \\
226 \text { propositions }\end{array}$ & $\begin{array}{c}5 \text { enfants } \\
274 \text { propositions }\end{array}$ & $\begin{array}{c}5 \text { enfants } \\
254 \text { propositions }\end{array}$ & \\
\hline 10 ans & $\begin{array}{c}5 \text { enfants } \\
297 \text { propositions }\end{array}$ & $\begin{array}{c}5 \text { enfants } \\
278 \text { propositions }\end{array}$ & $\begin{array}{c}5 \text { enfants } \\
260 \text { propositions }\end{array}$ & $\begin{array}{c}5 \text { enfants } \\
208 \text { propositions }\end{array}$ \\
\hline Adultes & 5 adultes 3 & & 2 adultes \\
& & & 206 propositions & \\
\hline
\end{tabular}

La section II étudie la distribution des modes d'organisation des énoncés 4 et l'emploi des connecteurs, dans les récits en langue française, la section III dans les récits en langue arabe, enfin la section IV compare les résultats obtenus dans les deux langues pour en tirer quelques conclusions interprétatives.

Les enfants développent progressivement des moyens de construire du discours de plus en plus cohésif, et nous examinons la complexification des énoncés dans cette perspective, en tant que dimension globale, appelée « condensation ». Les connecteurs en font partie puisqu'ils contribuent à effectuer la condensation, la diversifient et la rendent fonctionnellement plus flexible. Nous comparons l'acquisition et l'emploi de ces marqueurs pour caractériser la capacité de nos enfants à produire des récits en langue première, ou en deux langues dont l'une est moins maitrisée que l'autre. Enfin, nous examinons comment ces enfants confrontés à deux langues, typologiquement différentes du point de vue des procédés linguistiques de construction d'énoncés, mettent en relation les procès au sein d'énoncés mono- et pluripropositionnels dans leurs récits en chacune de ces langues.

\footnotetext{
2 «Est considérée comme une clause, toute unité qui contient un prédicat unifié. Par unifié, nous entendons un prédicat qui exprime une seule situation (activité, événement, état). Dans les prédicats, sont inclus les verbes fléchis et non fléchis ainsi que les adjectifs prédicatifs. En général, les clauses comprennent un seul élément verbal ; cependant, les infinitifs et les participes qui sont compléments d'un verbe modal ou aspectuel sont inclus à la matrice et donc considérés comme formant une seule clause » (Berman et Slobin, 1986).

3 Quatre des cinq enregistrements et transcriptions ont été faits par de Lorenzo Rossello, C. (2001a).

${ }^{4}$ Noyau (1991) choisit comme unité d'analyse pour étudier la temporalité dans les récits une unité cognitive, les situations, définies comme des états de choses dotés de localisation temporelle, représentés par des énoncés. La situation est aussi le contenu conceptuel de linterprétation contextuelle d'un énoncé (prise en compte de deux caractéristiques : l'une temporelle et l'autre énonciative).
} 
1. Organisation des énoncés et emploi des connecteurs dans les récits en français

\subsection{Organisation des énoncés}

Le tableau 2 illustre les définitions des énoncés que nous retenons (énoncés monopropositionnels, pluripropositionnels, paratactiques et hypotactiques).

Tableau 2 : Illustration d'énoncés mono / pluripropositionnels

\begin{tabular}{|c|c|c|c|c|}
\hline $\begin{array}{c}\text { Enoncés } \\
\text { monoproposi- } \\
\text { tionnels (ne } \\
\text { comportant } \\
\text { qu'une seule } \\
\text { proposition) }\end{array}$ & & & & $\begin{array}{l}\text { Un garçon et un chien regardent une } \\
\text { grenouille dans un bocal }\end{array}$ \\
\hline $\begin{array}{c}\text { Enoncés } \\
\text { pluriproposition- }\end{array}$ & Paratactiques & Asyndétique: & pause & $\begin{array}{l}\text { La grenouille a disparu on cherche } \\
\text { partout }\end{array}$ \\
\hline nels (comportant & & Syndétiques & & Il s'en va dans la forêt et il appelle \\
\hline & & & $\begin{array}{l}\text { Et renforcé (lien } \\
\text { faible) }\end{array}$ & $\begin{array}{l}\text { Il appelle dans un trou et puis le } \\
\text { chien il regarde }\end{array}$ \\
\hline $\begin{array}{l}\text { propositions } \\
\text { séparées soit par } \\
\text { une pause, soit } \\
\text { par un } \\
\text { connecteur) }\end{array}$ & & & $\begin{array}{c}\text { Conj.coord. } \\
\text { autres } \\
\text { Adv.liaison (lien } \\
\text { sémantiquement } \\
\text { plus riche) }\end{array}$ & $\begin{array}{l}\text { Le chien saute mais le bocal se casse } \\
\text { Le chien il aboie sur la ruche après } \\
\text { la ruche tombe } \\
\text { Un hibou le pousse alors il va autre } \\
\text { part }\end{array}$ \\
\hline & Hypotactiques & Pronoms relc & & $\begin{array}{l}\text { Il prend une grenouille qui vient de } \\
\text { naître }\end{array}$ \\
\hline & & Conjonction & que & $\begin{array}{l}\text { Il croit que la grenouille elle est } \\
\text { derrière un tronc d'arbre }\end{array}$ \\
\hline & & Subordonnan & circonst. & $\begin{array}{l}\text { Le chien tombe tandis que le petit } \\
\text { garçon appelle }\end{array}$ \\
\hline
\end{tabular}

Les résultats de notre étude peuvent être analysés tout d'abord selon deux axes, d'une part monopropositionnel / pluripropositionnel et d'autre part paratactique / hypotactique.

\section{Tableau 3:}

Enoncés monopropositionnels / pluripropositionnels (en pourcentage) dans les récits des enfants de notre étude.

(L'âge de 7 / 10 ans est précédé de la lettre F / B / A si monolinguisme LF / bilinguisme LF et LA / monolinguisme LA)

\begin{tabular}{|c|c|c|c|c|c|c|c|}
\hline $\begin{array}{c}\text { Enfants / } \\
\text { Adultes }\end{array}$ & $\begin{array}{c}\text { Mono- } \\
\text { Propos. }\end{array}$ & $\begin{array}{c}\text { Pluri- } 2 \\
\text { Propos. }\end{array}$ & $\begin{array}{c}\text { Pluri- 3 } \\
\text { Propos. }\end{array}$ & $\begin{array}{c}\text { Pluri- 4 } \\
\text { Propos. }\end{array}$ & $\begin{array}{c}\text { Pluri- 5 } \\
\text { Propos. }\end{array}$ & $\begin{array}{c}\text { Pluri- 6 } \\
\text { Propos. }\end{array}$ & $\begin{array}{c}\text { Pluri- } 7 \\
\text { Propos. }\end{array}$ \\
\hline F-7 & $37 \%$ & $40 \%$ & $19 \%$ & $2,5 \%$ & 0,5 & & \\
\hline F -10 & 30 & 34,5 & 27 & 6,3 & 0,7 & 1,5 & \\
\hline F-adultes & 16,8 & 32,9 & 24,8 & 13,7 & 4,9 & 4,4 & 2,5 \\
\hline B -7 & 56 & 36 & 8 & & & & \\
\hline B -10 & 49 & 39 & 10 & 2 & & & \\
\hline A -10 & 36,27 & 49,3 & 12,55 & 1,86 & & & \\
\hline
\end{tabular}


Les enfants de 10 ans et les adultes francophones natifs emploient moins d'énoncés monopropositionnels et d'énoncés à deux propositions dans les récits que les enfants de 7 ans francophones natifs, alors que les énoncés pluripropositionnels à plus de 5 propositions sont davantage utilisés chez les adultes. Par ailleurs, les enfants francophones natifs emploient davantage d'énoncés pluri-propositionnels à 3, 4 propositions (ou plus) que les enfants bilingues / apprenants de même âge respectif : constatation qui se retrouve lorsque l'on calcule le « taux de condensation », ou " le degré d'intégration-hiérarchisation de procès multiples en une seule énonciation » (rapport entre le nombre de propositions et le nombre d'énoncés) :

Tableau 4 : Rapport entre le nombre de propositions et le nombre d'énoncés

\begin{tabular}{|c|c|c|c|c|c|}
\hline $\mathrm{F}-7$ ans & $\mathrm{F}-10$ ans & $\mathrm{F}-$ Adultes & $\mathrm{B}-7$ ans & $\mathrm{B}-10$ ans & $\mathrm{A}-10$ ans \\
\hline 1,92 & 2,09 & 2,88 & 1,52 & 1,65 & 1,39 \\
\hline
\end{tabular}

On constate une augmentation de ce taux de condensation selon l'axe monopropositionnel / pluripropositionnel en fonction de l'âge, tant chez les monolingues francophones natifs que chez les enfants bilingues (F7/F10 et B7/B10).

Cependant, le fait de trouver des énoncés multipropositionnels ne signifie pas que l'hypotaxe soit privilégiée; en effet, on constate une prédominance d'énoncés à coordination dans les récits des enfants francophones de 7 ans, par et seul ou renforcé ${ }^{5}$ par après, ou puis :

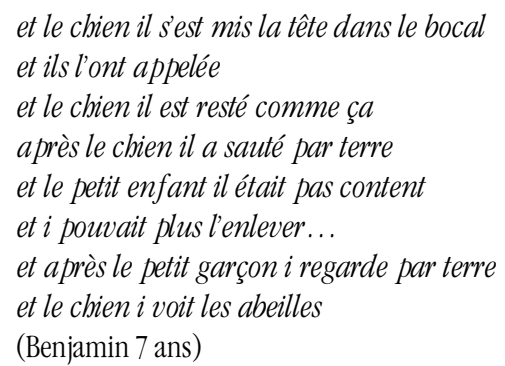

L'analyse des récits faite selon l'axe parataxe / hypotaxe donne les résultats suivants ${ }^{6}$.

Tableau 5 : Distribution des propositions paratactiques / hypotactiques

\begin{tabular}{|c|c|c|c|c|c|c|}
\hline & F-7ans & F-10 ans & B-7ans & B-10 ans & A-10 ans & F-Adultes \\
\hline Parataxe & $95 \%$ & $83 \%$ & $93 \%$ & $87 \%$ & $84 \%$ & $65 \%$ \\
\hline Hypotaxe & $\mathbf{5 \%}$ & $\mathbf{1 7 \%}$ & $7 \%$ & $13 \%$ & $16 \%$ & $\mathbf{3 5 \%}$ \\
\hline
\end{tabular}

\footnotetext{
${ }^{5}$ Le pourcentage de et renforcé par rapport au et non renforcé régresse de $30 \%$ dans des productions orales d'enfants n'ayant pas fréquenté l'école primaire à 6\% dans celles des enfants âgés de 7 ans (Sénémaud, M., 1997).

${ }^{6}$ Les résultats vont dans le même sens que ceux de Gayraud, F., Jisa H., Viguié, A., 2001.
} 
On constate que la parataxe est privilégiée par les plus jeunes enfants et que l'hypotaxe croît progressivement avec l'acquisition de structures syntaxiques plus complexes et le développement cognitif du locuteur.

bernard tombe de l'arbre
alors que justement le bibou
qui était caché dans le trou
dans lequel il cherchait sa grenouille
sort très rapidement d'un seul jet
et il le surprend (adulte)

Le tableau 6 ci-après comptabilise l'emploi des formes fléchies 7 / non fléchies du verbe dans les subordonnées :

il a mis la grenouille dans un pot quand il se couche (Clément 10 ans) vs il lui raconte une histoire avant de s'endormir (adulte)

Tableau 6 : Pourcentage de subordonnées à formes verbales fléchies $v s$ non fléchies

\begin{tabular}{|c|c|c|c|c|c|c|}
\hline & F-7ans & F-10 ans & B-7ans & B-10 ans & A-10 ans & $\begin{array}{c}\text { Adulte } \\
\text { Francophone }\end{array}$ \\
\hline F.V. fléchies & 65 & 78 & 60 & 79,4 & 71,4 & 77,6 \\
\hline F.V. non fléchies & 35 & 22 & 40 & 20,6 & 28,6 & 22,4 \\
\hline
\end{tabular}

Les subordonnées à formes verbales non fléchies semblent importantes par rapport aux subordonnées à formes verbales fléchies, mais elles sont peu nombreuses chez les enfants francophones natifs de 7 ans (35\% des 5\% de subordonnées), avec le schème « pour + V infinitif » : et puis le chien i disait ouaf ouaf pour chercher la grenouille.

Le tableau 7 présente le pourcentage des différentes catégories de propositions dans les productions en langue française des groupes francophones natifs et bilingues. Il permet de constater, pour les francophones natifs, que le pourcentage d'emploi des principales, subordonnées seules, emboîtées $^{8}$, enclavées ${ }^{9}$ et à verbe non fléchi augmente en fonction de l'âge (à la défaveur des indépendantes et de la coordination) : on retrouve donc les résultats de l'augmentation de l'hypotaxe en fonction de l'âge. Chez les bilingues B7 et B10, c'est le pourcentage des indépendantes coordonnées et des principales coordonnées qui augmente en fonction de l'âge.

\footnotetext{
${ }^{7}$ Cette catégorie correspond à "la subordination à verbe conjugué » (Koch, 1995) et comprend les conjonctions de subordination circonstancielle (quand, parce que, comme, pour que ...), les relatifs, les interrogatifs indirects, la conjonction que introduisant une complétive.

8 on pourra emmener un petit bébé grenouille qui pourra revoir sa famille quand il le voudra: proposition principale et deux propositions subordonnées dont une est emboîtée.

${ }^{9}$ le chien qui a toujours la tête dans le bocal tombe de la fenêtre: proposition principale et proposition subordonnée relative enclavée.
} 
Tableau 7 :

Pourcentage des différentes catégories de propositions par rapport au nombre total de propositions

\begin{tabular}{|c|c|c|c|c|c|c|c|c|c|}
\hline & \multirow{2}{*}{\multicolumn{2}{|c|}{$\begin{array}{l}\text { Propositions } \\
\text { indépendantes }\end{array}$}} & \multirow{2}{*}{\multicolumn{2}{|c|}{ Propositions principales }} & \multicolumn{5}{|c|}{ Propositions subordonnées } \\
\hline & & & & & \multicolumn{4}{|c|}{ à formes verbales fléchies } & \multirow[b]{2}{*}{$\begin{array}{c}\text { à formes } V \text {. } \\
\text { non } \\
\text { fléchies }\end{array}$} \\
\hline & $\begin{array}{c}\text { non } \\
\text { coordon- } \\
\text { nées }\end{array}$ & $\begin{array}{l}\text { coordon- } \\
\text { nées }\end{array}$ & $\begin{array}{l}\text { non } \\
\text { coordon- } \\
\text { nées }\end{array}$ & $\begin{array}{l}\text { coordon- } \\
\text { nées }\end{array}$ & seules & emboîtées & enclavées & coordonnées & \\
\hline F-7 ans & 27,4 & 56,6 & 3,1 & 4,9 & 4,5 & 0 & 0 & 0 & 2,65 \\
\hline F-10 ans & 29,9 & 34 & 6,1 & 5,7 & 15,8 & 0,7 & 0,67 & 1,68 & 5,39 \\
\hline F-Adultes & 29,89 & 20,43 & 7,31 & 7,74 & 22,15 & 2,58 & 1,08 & 1,08 & 7,74 \\
\hline $\mathrm{B}-7$ ans & 50 & 36,86 & 3,65 & 2,55 & 4,01 & 2,55 & 0,36 & 0 & 0 \\
\hline $\mathrm{B}-10$ ans & 20,14 & 57,55 & 1,82 & 8,99 & 8,99 & 1,80 & 0,36 & 0 & 0 \\
\hline$A-10$ ans & 35,48 & 40,72 & 6,85 & 2,82 & 8,87 & 0,8 & 0,4 & 0 & 4,03 \\
\hline
\end{tabular}

\title{
1.2. Fonctionnement et développement des connecteurs
}

La parataxe et l'hypotaxe sont réalisées avec ou sans connecteur, comme l'illustrent les exemples suivants :

\author{
on cherche à lintérieur de la botte \\ on cherche sous le lit dans le bocal (parataxe asyndétique ) \\ l'enfant voit \\ la grenouille elle est pas là (hypotaxe asyndétique, d'où forme idiosyncrasique)
}

Les connecteurs sont des outils de liaison, mots ou groupes de mots, permettant de relier des mots, des propositions ou des énoncés et de structurer un texte. Dans le récit, outre le connecteur additif et, il s'agit le plus souvent de connecteurs temporels, causaux, ou de but. Certaines expressions adverbiales en position initiale peuvent avoir fonction de connecteur: le lendemain matin tout le monde se réveille.

Les listes des connecteurs relevés dans les productions des quinze francophones natifs permettent déjà de rendre compte de quelques aspects de l'acquisition de la langue. La liste correspondant aux cinq enfants de 7 ans est très courte : essentiellement et, et renforcé par puis ou après, après, puis, alors, là, et quelques singletons : parce que, tout d'un coup, car, tandis que, où ( $\mathrm{y}$ a des abeilles), le matin. La liste relative au corpus des enfants de 10 ans conserve les éléments ci-dessus, en pourcentages différents, et s'enrichit d'autres: les conjonctions de temps quand, pendant que ; la conjonction de but pour que; la conjonction de concession / restriction sinon; les adverbes de temps ensuite, tout à coup; la conjonction de cause comme ; les coordonnants donc et mais; si à valeur d'interrogation indirecte; la préposition pendant; les expressions adverbiales en bas, le soir, le lendemain / lendemain matin.

La liste relative au corpus des cinq adultes s'enrichit des pronoms relatifs duquel, sur lequel, à laquelle, dont; des conjonctions et locutions conjonctives au cas où, puisque, tellement que, alors que; des expressions adverbiales par contre, ainsi et de l'expression spatiale au loin. 
Tableau 8: Enrichissement lexical et syntaxique selon l'âge (enfants bilingues et apprenants)

\begin{tabular}{|c|c|c|l|}
\hline Ages & B-7ans & B-10 ans & \multicolumn{1}{|c|}{ A- 10 } \\
\hline \multirow{3}{*}{ Occurrences des } & et / et après / et puis/ & et encore / aussi & et / mais / après / puis / \\
connecteurs & et aussi / après / & encore / alors / donc & mais / alors après / \\
& après aussi / & comme / quand / qui / & soudain / tout à coup/ \\
& comme ça & que / où & quand / \\
& mais / pour / & sauf que / pareil que / & pour / parce que / que \\
& si / quiest-ce que & une fois dans la nuit / & \\
& pour que / parce que & un jour / la nuit / & \\
& tout à coup / soudain/ & le lendemain matin / & \\
& le matin & le soir & \\
\hline
\end{tabular}

Tableau 9:

Pourcentages significatifs de quelques mots de liaison par rapport au nombre de propositions

\begin{tabular}{|c|c|c|c|c|c|c|c|c|c|c|}
\hline Liens & Et & Après & $\begin{array}{c}\text { Et } \\
\text { renforcé }\end{array}$ & Puis & $\begin{array}{c}\text { Là } \\
\text { (déictique) }\end{array}$ & Mais & Donc & $\begin{array}{c}\text { Adverbes } \\
\text { de temps } \\
\text { autres }\end{array}$ & $\begin{array}{c}\text { (il) y a } \\
\text {..qui } / \\
\text { c'est } \\
\text {..qui }\end{array}$ & $\begin{array}{c}\text { Pronoms } \\
\text { relatifs }\end{array}$ \\
\hline F-7 ans & 28,32 & 19,03 & 13,27 & 8,6 & 6,9 & 0,66 & 1,97 & 3,53 & 3,98 & 2,65 \\
\hline F-10 ans & 24,24 & 6,73 & 2,69 & 4 & 3,4 & 1,68 & 3,03 & 4,38 & 1,69 & 4,04 \\
\hline F- adultes & 20,27 & 1,34 & 0 & 0,9 & 0 & 2,67 & 3,79 & 8,02 & 0,45 & 11,8 \\
\hline B- 7 ans & 29,19 & 8,02 & 2,89 & & & 1,82 & & 3,65 & 0,36 & 1,5 \\
\hline B-10 ans & 34,53 & 21,53 & 4,28 & & & 0,71 & 1,43 & 8,63 & 2,52 & 2,2 \\
\hline A-10 ans & 15,88 & 2,33 & & 0,46 & & & 8,87 & & 0,46 & \\
\hline
\end{tabular}

Le tableau 9 fait apparaitre que le nombre des occurrences de et, et renforcé, après, puis, là (déictique) diminue avec l'âge, et que celui des occurrences de mais, donc, et autres adverbes de temps augmente pour le groupe des francophones natifs.

Même si l'on ne doit pas oublier l'influence de la scolarisation, donc de l'écrit, dans l'oralisation, on peut observer une certaine évolution de la complexité des énoncés: davantage d'énoncés à lien hypotactique, d'énoncés avec plus de trois propositions, le remplacement de et par d'autres connecteurs sémantiquement plus forts ${ }^{10}$, propositions à verbes non fléchis, apparition de la nominalisation :

le lendemain ills) voient que la grenouille n'est plus dans le pot $\quad$ (Clément 10 ans) le lendemain matin surprise absolument plus rien dans le bocal la grenouille s'est envolée

ensuite ils prennent la direction du bois tous les deux

(adulte)

et toujours à la recherche de la grenouille (adulte)

et de la gérondivisation :

c'est en courant près de l'arbre quil renverse le petit garçon (adulte).

Nous retrouvons là la même évolution que celle décrite pour les apprenants adolescents polonophones de français (Noyau \& Paprocka, 2000), en trois étapes :

\footnotetext{
10 mais et donc véhiculant respectivement une idée d'opposition et de consécution peuvent être considérés comme connecteurs sémantiquement plus forts que $e t$.
} 


\section{Etape I}

La parataxe est dominante.

La proportion propositions / énoncés n'est pas très élevée.

Les connecteurs les plus fréquemment rencontrés sont et, et puis, et après, y a ...qui, c'est ...qui, que (dit que).

Quelques complétives, des relatives avec présentatif, quelques relatives,

pas ou peu de subordonnées de cause ou de but.

\section{Etape II}

La proportion propositions / énoncés est supérieure à la précédente.

Des connecteurs de consécutivité comme puis, ensuite, alors, apparaissent.

Les énoncés hypotaxiques émergent sélectivement dans des contextes spécifiques.

La construction de type moyen-but se fait avec une infinitive.

\section{Etape III}

La proportion propositions / énoncés est élevée.

Il y a davantage de structures relationnelles et de connecteurs « forts ».

Dans les énoncés pluripropositionnels, on trouve des propositions au même niveau hiérarchique (parataxe),

mais aussi des énoncés hypotactiques avec des connecteurs explicites de mise en dépendance

d'une proposition, ou de plusieurs.

\section{Construction des récits et connecteurs en arabe tunisien}

Avant de présenter les résultats obtenus à partir des productions de nos apprenants issus de l'immigration en arabe, nous analysons le comportement langagier d'un groupe témoin adultes, de façon à rendre possible la pondération des facteurs cognitifs et des facteurs linguistiques dans l'évolution de la maîtrise de la hiérarchisation et de la planification du discours ${ }^{11}$.

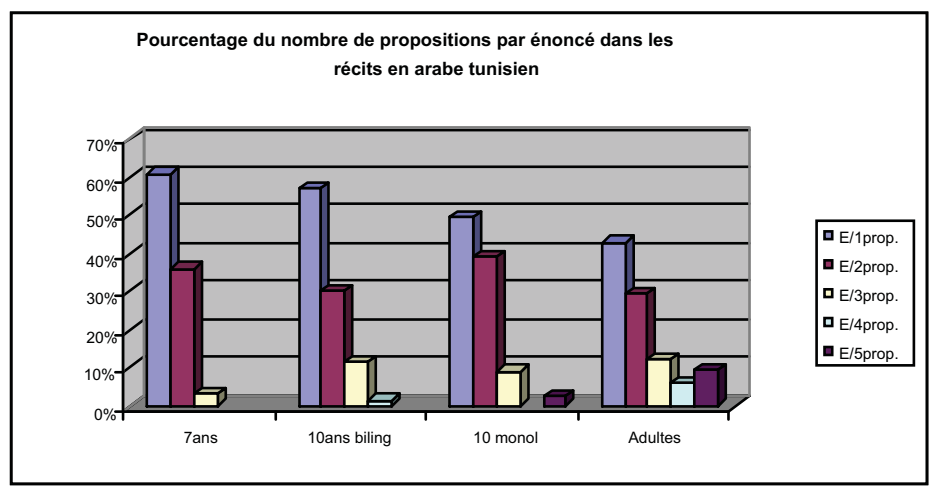

Figure 1

\footnotetext{
${ }^{11}$ Les comparaisons en arabe avec les enfants monolingues tunisiens ne seront faites qu'entre les groupes de 10 ans, étant donné que les enfants tunisiens ne commencent l'apprentissage du français qu'à l'âge de 8 ans.
} 
On l'a vu (section 1), le taux d'énoncés pluripropositionnels connaît un développement des plus jeunes aux adultes chez les bilingues en France. Comme dans les productions en français, les plus jeunes peinent ici à se détacher du support et construisent plutôt des énoncés monopropositionnels (61\%) et à deux propositions (36\%). Chez les 10 ans, on remarque un développement des énoncés à trois propositions (12\%), et l'émergence des énoncés à quatre propositions (2\%). Ce développement de la condensation peut être mis en rapport, d'une part, avec l'exposition à la langue arabe ${ }^{12}$, et d'autre part, avec l'environnement didactique ${ }^{13}$. Chez les adultes, le taux des énoncés manifestant de la condensation est beaucoup plus élevé (57\%), mais le pourcentage des énoncés monopropositionnels reste important (43\%), confirmant la tendance à la parataxe en arabe tunisien.

\subsection{Parataxe - Hypotaxe}

Nous avons constaté la répartition suivante des moyens de la parataxe et de l'hypotaxe chez nos sujets arabophones :

Tableau 10 : Distribution des propositions paratactiques / hypotactiques

\begin{tabular}{|c|c|c|c|}
\hline Enfants & PARATAXE & HYPOTAXE & Nombre de propositions \\
\hline B-7ans & $94 \%$ & $6 \%$ & 254 \\
\hline B-10ans & $91 \%$ & $9 \%$ & 260 \\
\hline A-10 ans & $88,73 \%$ & $11,27 \%$ & 204 \\
\hline Adultes & $74 \%$ & $26 \%$ & 206 \\
\hline
\end{tabular}

Globalement, les quatre groupes manifestent une prédominance de la parataxe, qui diminue, néanmoins, avec l'âge, au profit de l'hypotaxe. Les enfants privilégient souvent la parataxe là où les adultes emploient une construction hypotactique comme dans les exemples suivants ${ }^{14}$ :

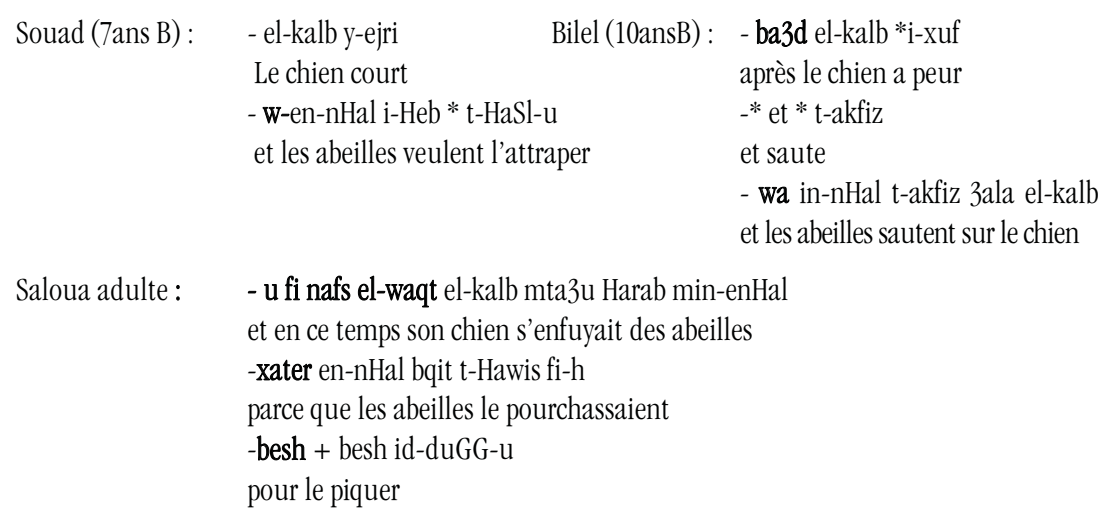

12 Influence de l'usage de l'arabe par l'adulte (surtout la mère) et des programmes de la télévision tunisienne (captés grâce aux paraboles).

13 Selon le programme d'arabe à l'école bilingue de la rue de Tanger, à Paris $19^{\text {ème }}$, en CM1 et en CM2, le travail est centré sur la construction de la phrase complexe.

${ }^{14}$ Les membres arabisants de l'équipe « Acquisition » de Paris X ont adopté un code de transcription phonologique composé, à deux exceptions près, de caractères latins. 
Par ailleurs, les adultes emploient des constructions beaucoup plus diversifiées (parataxe syndétique et hypotaxe), avec une diversité formelle et fonctionnelle des connecteurs :

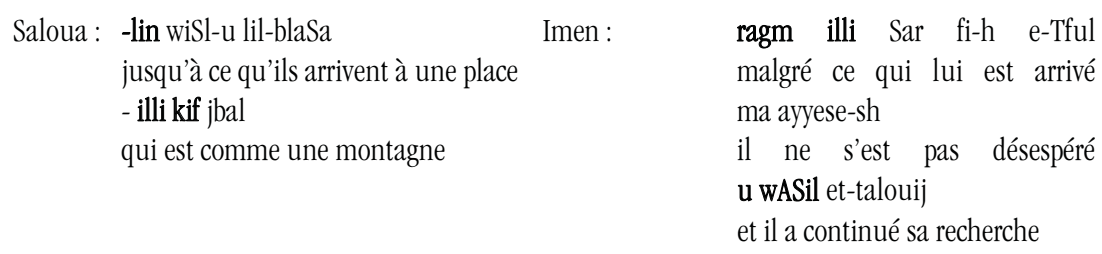

\subsubsection{Parataxe: Asyndèse / Syndèse}

Contrairement aux récits du groupe 10 ans bilingues, chez qui la parataxe syndétique (63\%) est beaucoup plus fréquente que l'asyndétique (29\%), celle-ci reste prédominante chez les 7 ans : $56 \%$ des propositions. Cela peut être relié non seulement à l'usage de l'arabe tunisien, où les liens entre propositions et la succession des événements sont facilement implicites [d'où la proportion asyndèse / syndèse équilibrée chez les adultes (39\% asyndèse et 35\% syndèse)], mais aussi au fait que les plus jeunes n'ont pas encore la capacité de gérer une tâche cognitivement lourde (planifier un discours long), ni à enchaîner explicitement les énoncés avec des liens spécifiques ${ }^{15}$. On voit également que la syndèse chez les B10 (63\%) est légèrement plus présente que chez les monolingues de même âge (62\%) : Cela relève non pas d'une parfaite maitrise des moyens syndétiques (dont l'emploi est parfois idiosyncrasique) mais plutôt de l'influence de leur deuxième langue : le français, où à l'oral les enfants ont tendance à sur-utiliser et, après, pris, et après, et puis... pour faire avancer leur discours.

Sara Zem :

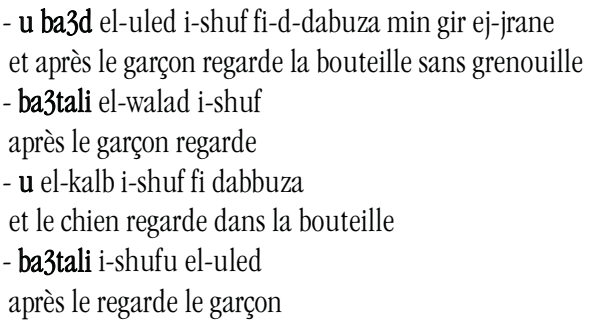

\subsubsection{Hypotaxe}

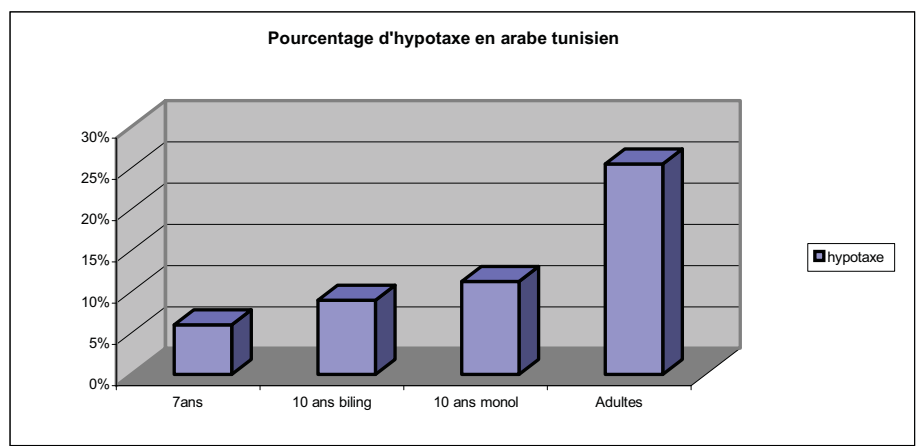

Figure 2

15 Allal, 1999 et thèse en cours. 
La figure 2 montre une évolution dans l'hypotaxe des plus jeunes aux adultes $(5 \%, 9 \%$, $11,27 \%, 28,1 \%$ ). Ainsi, la structure relationnelle dans un énoncé complexe peut être rendue avec un degré de hiérarchisation important, quand les moyens linguistiques sont disponibles chez le locuteur.

\subsection{Les liaisons syntaxiques}

\subsubsection{La parataxe : juxtaposition / coordination}

Tableau 11 : Pourcentages de quelques mots de liaison par rapport au nombre de propositions

\begin{tabular}{|c|c|c|c|c|c|c|c|c|}
\hline Groupes & wa/u (et) & $\begin{array}{c}\text { ba3d } \\
\text { (après) }\end{array}$ & $\begin{array}{c}\text { wa / u ba3d } \\
\text { (et après) }\end{array}$ & $\begin{array}{c}\text { Hatta/ zeda } \\
\text { (aussi) }\end{array}$ & $\begin{array}{c}\text { u zeda / u } \\
\text { Hatta } \\
\text { (et aussi) }\end{array}$ & yaxi (alors) & 3ada (donc) & amma (mais) \\
\hline B-7ans & $24,41 \%$ & $9 \%$ & $4 \%$ & $0 \%$ & $1 \%$ & $0,77 \%$ & $0 \%$ & $0 \%$ \\
\hline B-10 ans & $38,85 \%$ & $8,46 \%$ & $17,30 \%$ & $0,77 \%$ & $2,69 \%$ & $2,36 \%$ & $0 \%$ & $0 \%$ \\
\hline A-10 ans & $27 \%$ & $0 \%$ & $0 \%$ & $0,49 \%$ & $0 \%$ & $18 \%$ & $9,80 \%$ & $0,48 \%$ \\
\hline Adultes & $20,87 \%$ & $1,45 \%$ & $0,48 \%$ & $0,48 \%$ & $0 \%$ & $5,34 \%$ & $1 \%$ & $1 \%$ \\
\hline
\end{tabular}

La condensation des informations au niveau conceptuel se manifeste au niveau de la formulation linguistique par l'emploi des outils syntaxiques marquant des liens interpropositionnels. Comme nous l'avons vu, la parataxe asyndétique est majoritaire chez les enfants de 7 ans. Leurs récits, linéaires, comportent peu d'énoncés complexes, et les relations entre les propositions sont le plus souvent implicites. Les moyens explicites relevés dans leurs récits se résument aux coordonnants wa 'et', ba3d 'après', u ba3d 'et après', pluri-fonctionnels (fonction additive, consécutivité).

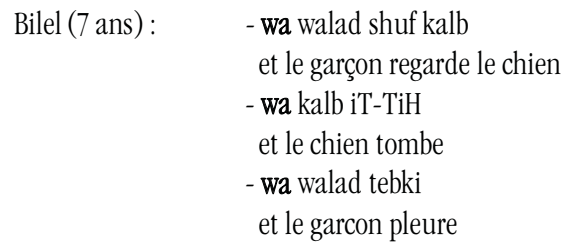

Les enfants bilingues plus âgés recourent davantage à $w a$ 'et', $m b a 3 d$ 'après', $u$ mba3d 'et après' que les autres groupes. Aux fonctions additive et de consécutivité s'ajoutent parfois des liens causaux et de concomitance avec wa Hatta 'et aussi' pour décrire les actions parallèles du garçon et de son chien.

$$
\begin{array}{ll}
\text { Rafika (10): } \quad \text { - u Hatta i-Tufl huwa i-nAdi a3li-ha } & \text { Et aussi le garcon l'appelle }
\end{array}
$$

L'emploi de ces connecteurs est parfois superflu, ce qui peut être la conséquence de l'influence du français oral, où les enfants emploient fréquemment et sans spécialisation sémantique et, et après, et puis.

Enfin, chez les enfants monolingues et les adultes de Tunisie, nous remarquons un moindre recours aux coordonnants à valeur temporelle que les bilingues. Ces deux groupes de locuteurs dont l'arabe est la langue forte disposent d'un éventail de formes linguistiques plus large permettant l'expression de relations sémantiques diverses et la hiérarchisation, avec les subordonnants $k i$ 'quand', Hatta lin 'jusqu'à ce que', ba3d ma 'après que', ainsi que yaxi, 3ada 'alors / donc' et 
amma 'mais' exprimant des relations de conséquence et d'adversativité. Ces connecteurs permettent de structurer les discours de manière globale en articulant des épisodes. Enfin, des expressions temporelles telles que $f$-il-lil 'le soir', $f$-iSbAH 'le matin', aux charnières d'épisodes, servent à structurer les récits et jouent un rôle de connecteurs, dans toutes les productions.

\subsubsection{Hypotaxe: subordonnées à formes verbales fléchies / subordonnées à formes verbales non fléchies}

Le tableau 12 montre une évolution dans l'emploi des structures complexes et dans les fonctions communicatives des subordonnées. Etant au début de leur apprentissage de la langue arabe, les enfants de 7 ans trouvent plus de difficultés à hiérarchiser les informations et à les agencer dans des constructions complexes. Dans leurs productions émergent quelques subordonnées spécifiant des relations sémantiques de cause, de conséquence et de but. La liste des subordonnées s'enrichit chez les enfants de 10 ans bilingues d'autres types comme la subordonnée de manière, la relative, et l'interrogative indirecte dont l'usage n'est pas fréquent en arabe tunisien. En effet, le locuteur arabophone préfère l'interrogation directe, ce que montre la quasi-absence de cette construction chez les monolingues et les adultes.

Tableau 12:

Nombre des subordonnées à formes verbales fléchies / non fléchies dans les récits en arabe

\begin{tabular}{|c|c|c|c|c|c|c|c|c|c|c|c|c|c|c|c|}
\hline & \multicolumn{12}{|c|}{ subordonnées à formes verbales fléchies } & \multicolumn{3}{|c|}{$\begin{array}{l}\text { subordonnées à F.V. non } \\
\text { fléchies }\end{array}$} \\
\hline & \multicolumn{2}{|c|}{ relatives } & \multirow{2}{*}{$\begin{array}{l}\text { Com- } \\
\text { plétives }\end{array}$} & \multirow{2}{*}{$\begin{array}{c}\text { Interro- } \\
\text { gatives } \\
\text { indirectes }\end{array}$} & \multicolumn{8}{|c|}{ circonstancielles } & \multirow{2}{*}{$\begin{array}{l}\text { besh } \\
+ \\
\text { ffléchie }\end{array}$} & \multirow{2}{*}{$\begin{array}{l}\text { Parti- } \\
\text { cipe } \\
\text { actif } \\
\end{array}$} & \multirow{2}{*}{$\begin{array}{l}\text { Parti- } \\
\text { cipe } \\
\text { passif } \\
\end{array}$} \\
\hline & $\begin{array}{l}\text { illi } \\
\text { (qui) }\end{array}$ & ce qui & & & temps & cause & $\operatorname{csq}$ & $\begin{array}{l}\text { Compa- } \\
\text { raison }\end{array}$ & $\begin{array}{l}\text { Ma- } \\
\text { nière }\end{array}$ & $\begin{array}{l}\text { Conoes- } \\
\text { sion }\end{array}$ & $\begin{array}{l}\text { Condi- } \\
\text { tion }\end{array}$ & $\begin{array}{l}\text { Restric- } \\
\text { tion }\end{array}$ & & & \\
\hline $\begin{array}{l}\text { B-7 } \\
\text { ans }\end{array}$ & & 2 & & 1 & & 7 & 1 & & & & & & 1 & & \\
\hline $\begin{array}{l}\mathrm{B}-10 \\
\text { ans }\end{array}$ & 1 & & & 6 & & 2 & 1 & & 1 & & & & 5 & & \\
\hline $\begin{array}{c}\mathrm{A}-10 \\
\text { ans }\end{array}$ & 1 & & 1 & 0 & 12 & 1 & & & 1 & & & & 13 & & \\
\hline Adultes & 4 & & 1 & 1 & 6 & 3 & 2 & 1 & 6 & 1 & 1 & 1 & 10 & 7 & 3 \\
\hline
\end{tabular}

Le tableau 13 indique les moyens explicites employés dans les récits des quatre groupes.

Tableau 13: Les subordonnants employés dans les productions en arabe tunisien

\begin{tabular}{|c|c|c|c|c|c|c|c|c|c|c|c|c|c|}
\hline & \multicolumn{13}{|c|}{ movens explicites employés dans les récits en arabe } \\
\hline & \multirow{2}{*}{\multicolumn{2}{|c|}{ relatives }} & \multirow{2}{*}{$\begin{array}{l}\text { Complé- } \\
\text { tives }\end{array}$} & \multirow{2}{*}{$\begin{array}{l}\text { Interro- } \\
\text { gatives } \\
\text { indirectes }\end{array}$} & \multicolumn{9}{|c|}{$\begin{array}{cc} & \text { circonstancielles } \\
\end{array}$} \\
\hline & & & & & temps & cause & $\begin{array}{l}\text { Consé- } \\
\text { quence }\end{array}$ & $\begin{array}{l}\text { Compa- } \\
\text { raison }\end{array}$ & $\begin{array}{l}\text { Ma- } \\
\text { nière }\end{array}$ & $\begin{array}{c}\text { Conces- } \\
\text { sion }\end{array}$ & $\begin{array}{c}\text { Condi- } \\
\text { tion }\end{array}$ & but & $\begin{array}{l}\text { Restric- } \\
\text { tion }\end{array}$ \\
\hline $\begin{array}{l}\text { B-7 } \\
\text { ans }\end{array}$ & & ce qui & & win & & $\begin{array}{c}\text { xater } \\
\text { parce } \\
\text { que }\end{array}$ & $\begin{array}{c}\text { comme } \\
\text { ça }\end{array}$ & & & & & $\begin{array}{l}\text { besh + } \\
\text { f. préf }\end{array}$ & \\
\hline $\begin{array}{c}\text { B- } \\
10 \\
\text { ans }\end{array}$ & $\begin{array}{c}\text { illi } \\
\text { (qui) }\end{array}$ & & & win & & $\begin{array}{c}\text { xater } \\
\text { 3ala } \\
\text { xater }\end{array}$ & $\begin{array}{c}\text { comme } \\
\text { ça }\end{array}$ & & hakka & & & $\begin{array}{l}\text { besh + } \\
\text { f. préf }\end{array}$ & \\
\hline $\begin{array}{c}\text { A- } \\
10 \\
\text { ans }\end{array}$ & $\begin{array}{l}\text { elli } \\
\text { win }\end{array}$ & & & win & $\begin{array}{c}\mathrm{ki} \\
\text { 3ada } \\
\mathrm{ki} \\
\mathrm{Hatta} \\
\text { lin } \\
\text { Waqt } \\
\text { Mb3d } \\
\mathrm{ma}\end{array}$ & xater & & & & & & $\begin{array}{l}\text { besh + } \\
\text { f. préf }\end{array}$ & ma gir \\
\hline $\begin{array}{l}\text { Adul- } \\
\text { tes }\end{array}$ & illi & & $\begin{array}{l}\text { illi } \\
\text { (que) }\end{array}$ & win & $\begin{array}{l}\text { i\$a bi } \\
\text { Hatta } \\
\text { lin } \\
\text { lin } \\
\text { ki }\end{array}$ & $\begin{array}{c}\text { xater } \\
\text { 3ala } \\
\text { inu }\end{array}$ & hakkaka & kif & $\begin{array}{c}\text { hakka } \\
\text { kima }\end{array}$ & ragm illi & killi & $\begin{array}{l}\text { besh + } \\
\text { f. préf }\end{array}$ & min gir \\
\hline
\end{tabular}


Les subordinations circonstancielles augmentent en fonction de l'âge et du niveau d'acquisition de la langue. La diversité des outils employés (et des relations sémantiques qu'ils encodent) se développe depuis 7 ans jusqu'à l'âge adulte, signe d'un développement au niveau interpropositionnel et au niveau du texte (hiérarchisation et planification). Cette évolution se fait en fonction de l'exposition à la langue (l'arabe tunisien en Tunisie $v s$ l'arabe tunisien en France). En effet, seuls les enfants monolingues et surtout les adultes emploient des subordonnants temporels (ki, waqtelli, Hatta lin...). Les enfants issus de l'immigration se contentent de constructions paratactiques avec wa, ba3d, u mba3d... pour établir des liens temporels entre les événements.

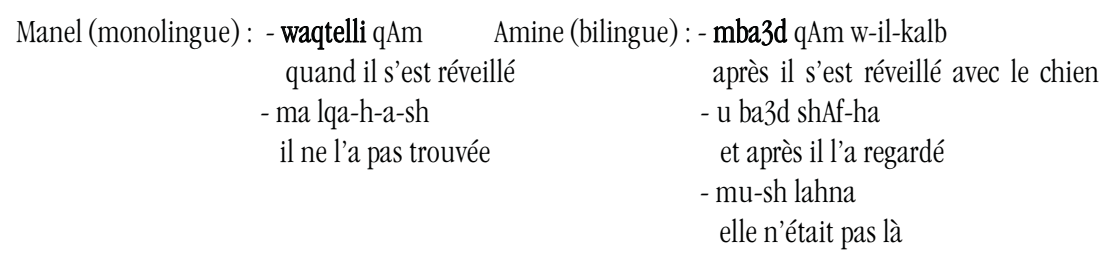

Pour le reste, les relations de cause introduites par xater et son équivalent français parce que sont les plus présentes chez les plus petits ${ }^{16}$. Les 10 ans bilingues utilisent surtout les subordinations de manière avec bakka et de but avec le 'lexème grammaticalisé' (cf. Brahim, 1997) besh suivi d'un verbe à la forme préfixale. Leur emploi de win 'où' marquant une interrogation indirecte est la conséquence de l'influence du français, étant donné qu'en arabe on privilégie l'interrogation directe. Les complétives avec illi 'que' sont surtout relevées chez les monolingues et les adultes. L'emploi des relatives est très faible chez les bilingues contrairement aux monolingues (deux relatifs français sans antécédent chez les plus jeunes et un relatif sujet illi 'qui' chez leurs aînés).

On relève également, dans le tableau ci-dessus, un éventail plus riche de formes et de fonctions des subordonnants chez les adultes, l'organisation de leurs récits étant marquée par une hiérarchisation et une planification des événements en unités informationnelles plus larges: des constructions hypotactiques à connecteurs comparatifs, hypothétiques, restrictifs et concessifs, ainsi que des propositions à participe actif et des participes passifs spécifiant des actants ou la manière des procès.

$$
\begin{aligned}
& \text { Imen : - w-el-kalb xAyef Imen : - w-el-kalb mta3-u mafju3 } \\
& \text { et le chien qui a eu peur / ayant peur et son chien qui est terrifié / étant terrifié } \\
& \text { - Hab y-aTla3 f-eshejra - y-ejri fi jurr-it el-gzala } \\
& \text { (il) voulait monter sur l'arbre il court après la gazelle }
\end{aligned}
$$

A côté de cette capacité à entretenir plusieurs niveaux de hiérarchisation dans un même énoncé (émergence de quelques subordonnées chez les bilingues, diversification des moyens chez les monolingues), nous constatons une prédominance de la parataxe (syndétique et asyndétique) dans les deux plans discursifs (trame / arrière-plan). Au plan syntaxique, les énoncés polypropositionnels à condensation faible sont dominants. Cela peut s'expliquer par le fait que, en arabe tunisien, on privilégie la parataxe dans l'organisation de l'information : les liens entre propositions sont souvent non marqués et laissés aux inférences pragmatiques (cf. Brahim, 1997). La condensation n'implique pas forcément la dominance de l'hypotaxe.

\footnotetext{
16 Dans cette tranche d'âge, on relève également une consécutive introduite par l'expression française comme ça.
} 


$$
\begin{aligned}
& \text { Sara Gui (7ans) : - el-kalb y-ejri Sonia (10ans) : - irkib fuG el ++ (comment on dit rocher?) } \\
& \text { le chien court el-Hajra } \\
& \text { - en-nHal + en-nHal y-ejr-u a3li-h il est monté sur le rocher } \\
& \text { les abeilles le poursuivent } \quad \text { - i-nAdi f-il }+\mathrm{f}-\mathrm{il}++ \text { ej-jrana } \\
& \text { il appelle la grenouille. }
\end{aligned}
$$

Entre les deux propositions de même statut énonciatif, dans ces exemples, existe un lien d'implication non marqué linguistiquement qui peut être interprété pragmatiquement en relation de cause à effet pour le premier exemple et en relation de but pour le second.

En résumé, au niveau global du façonnage des informations, la condensation est favorisée surtout chez les monolingues et les adultes. C'est la juxtaposition, donc une cohésion purement locale, qui est privilégiée dans notre corpus, dans l'usage oral de l'arabe tunisien.

\section{Comparaison des récits en français et en arabe}

Tableau 14 : Degré de condensation des énoncés en L.F. et L.A.

\begin{tabular}{|c|c|c|}
\hline Groupes & condensation en LF & condensation en LA \\
\hline 7 ans & 1,52 & 1,24 \\
\hline 10 ans bil. & 1,65 & 1,36 \\
\hline 10 ans monol. & & 1,38 \\
\hline Adultes & & 1,53 \\
\hline
\end{tabular}

Les récits en L.F. et en L.A. ${ }^{17}$ révèlent un certain degré de condensation. L'organisation de l'information se caractérise par une progression dans la planification : présence d'énoncés allant de 2 à 4 propositions dans les deux langues. La comparaison des résultats permet d'avancer les remarques suivantes :

- la capacité à produire des énoncés complexes est plus développée en L.F. : cela révèle un niveau plus avancé en français chez les bilingues ;

- elle est plus importante chez le groupe de 10 ans. Nous avons plus affaire, chez les plus jeunes, à des descriptions d'images qu'à de véritables narrations. Ils ne maîtrisent pas encore toutes les fonctions des outils qu'ils utilisent et, surtout, ne savent pas encore exploiter les connecteurs au profit de la cohérence. L'influence de l'école (donc de l'apprentissage de l'arabe littéral écrit ${ }^{18}$ ) s'exerce sur l'usage de l'oral (l'arabe tunisien).

Tableau 15 : Parataxe / hypotaxe en L.F. et en L.A.

\begin{tabular}{|c|c|c|c|c|}
\cline { 2 - 5 } \multicolumn{1}{c|}{} & \multicolumn{2}{c|}{ Parataxe } & \multicolumn{2}{c|}{ Hypotaxe } \\
\hline Enfants & L.F & L.A. & L.F. & L.A. \\
\hline 7ans & $93 \%$ & $94 \%$ & $7 \%$ & $6 \%$ \\
\hline 10 ans biling. & $88 \%$ & $91 \%$ & $13 \%$ & $9 \%$ \\
\hline 10 ans monol. & $83,65 \%$ & $88,72 \%$ & $16,35 \%$ & $11,27 \%$ \\
\hline Adultes & & $71,9 \%$ & & $28,1 \%$ \\
\hline
\end{tabular}

\footnotetext{
17 Afin d'éviter tout malentendu engendré par la complexité de la situation des enfants de l'immigration en France (quelle serait leur L1 et leur L2 ?), nous avons opté pour une terminologie neutre : la langue arabe serait leur LA et la langue française leur LF.

18 En CM1 les élèves apprennent la construction de la phrase complexe.
} 
Tableau 16 : Parataxe : asyndèse / syndèse

\begin{tabular}{|l|r|r|r|r|}
\cline { 2 - 5 } \multicolumn{1}{c|}{} & \multicolumn{2}{c|}{$\begin{array}{c}\text { Parataxe } \\
\text { Asyndétique }\end{array}$} & \multicolumn{2}{c|}{$\begin{array}{c}\text { Parataxe } \\
\text { syndétique }\end{array}$} \\
\hline Enfants & L.F & L.A. & L.F. & \multicolumn{1}{c|}{ L.A. } \\
\hline 7 ans & $49 \%$ & $56 \%$ & $44 \%$ & $39 \%$ \\
\hline 10 ans biling. & $19 \%$ & $29 \%$ & $69 \%$ & $63 \%$ \\
\hline 10 ans monol. & $44,39 \%$ & $26,96 \%$ & $39,25 \%$ & $61,76 \%$ \\
\hline Adultes & & $40,3 \%$ & & $31,6 \%$ \\
\hline
\end{tabular}

Tableau 17 : Hypotaxe : subordonnée fléchie / subordonnée non fléchie

\begin{tabular}{|c|c|c|c|c|}
\cline { 2 - 5 } \multicolumn{1}{c|}{} & \multicolumn{4}{c|}{ Hypotaxe } \\
\cline { 2 - 5 } \multicolumn{1}{c|}{} & \multicolumn{3}{c|}{ sub. F. } & sub. non F. \\
\hline Groupes & L.F. & L.A. & L.F. & L.A. \\
\hline 7 ans & $60 \%$ & $92 \%$ & $40 \%$ & $8 \%$ \\
\hline 10 ans biling. & $79 \%$ & $68,75 \%$ & $21 \%$ & $31,25 \%$ \\
\hline 10 ans monol. & $71,42 \%$ & $60,86 \%$ & $28,57 \%$ & $39,13 \%$ \\
\hline Adultes & & $58 \%$ & & $42 \%$ \\
\hline
\end{tabular}

Globalement, les résultats révèlent un privilège de l'organisation linéaire (la juxtaposition) au détriment des procédés syntaxiques en L.A. (tabl. 16). En revanche, surtout chez les plus âgés, les moyens syntaxiques (particulièrement ceux de l'hypotaxe) sont plus employés en L.F. Cette capacité à manier des structures complexes indique un niveau de maitrise discursive plus avancé. Les capacités de planification se développent quand les moyens linguistiques deviennent disponibles chez le locuteur.

Au niveau de la formulation, on note :

- une différence entre les productions en français et les productions en arabe tunisien chez

- les bilingues, les premières sont structurées au moyen d'une diversité lexicale de connecteurs (après, et après, ensuite, mais, pour, pour que, quand, comme ça, que, qui, sauf que, pareil que, si, où...) plus grande que les secondes, qui sont organisées par un nombre plus limité de connecteurs, parfois empruntés à la langue française (mba3d, yaxi, xater, win, parce $q u e . .$.$) . Ces enfants ont une organisation plus hiérarchisée du discours en L.F., par le recours aux$ fonctions sémantiques variées de la subordination, ce qui peut être rapporté à deux facteurs :

- les enfants, ayant un niveau plus avancé en français, ont acquis un éventail de connecteurs en L.F. plus important qu'en L.A. (facteur acquisitionnel),

- l'arabe est une langue où certaines relations logico-sémantiques entre les propositions sont effectuées de manière implicite à travers le contexte (facteur typologique).

- une capacité à produire des énoncés complexes plus évoluée chez les monolingues arabophones qui, contrairement aux bilingues, ont à leur disposition un éventail varié de formes dont ils maîtrisent les différentes fonctions. Ils encodent, surtout les adultes, des relations temporelles entre les événements, et différentes relations logiques. En revanche, les productions des bilingues sont organisées selon une structure plus élémentaire, avec une hiérarchisation moindre entre les événements, indiquant un niveau de maitrise discursive moins élevé que les monolingues, ce qui peut être rapporté au degré d'exposition à la langue arabe. Les enfants de familles migrantes qui 
vivent en France trouvent des difficultés à apprendre l'arabe tunisien. Ces difficultés s'atténuent avec l'apprentissage de l'arabe littéral à l'école, mais cette variété de l'arabe est loin d'être pratiquée au quotidien.

\section{Conclusion}

Nos résultats comparatifs montrent plusieurs convergences intéressantes avec des ensembles de travaux de psychologie expérimentale sur l'acquisition de la capacité narrative orale et écrite en français chez les enfants :

- les enfants de 7 ans préêrent les enchaînements paratactiques neutres avec $e t$, alors que ceux de 10 ans recourent aux connecteurs sémantiquement différenciés, surtout temporels, lorsqu'ils s'agit de transitions entre macro-événements (passage entre images successives); on rend compte de cette évolution par l'interaction entre deux développements interdépendants: du point de vue cognitif, l'accroissement de la capacité à adopter un traitement global 'descendant', du point de vue linguistique, la maîtrise du système des connecteurs (Favart \& Passerault 1995);

- la production de récits oraux met en évidence des capacités qui ne sont présentes que chez des enfants plus âgés dans les productions écrites : ce retard s'explique par le fait que l'accroissement de la difficulté de traitement sur un paramètre ou un autre dans une tâche cognitive complexe telle que celle de construire un récit ramène le locuteur à un mode de traitement plus linéaire, superficiel et dépendant du support (Bourdin \& Fayol 1994, Fayol 2000).

Le fait de construire un récit dans une langue étrangère, ou dans la langue 'faible' chez les bilingues (Schlyter 1995) est-il un facteur similaire de difficulté du traitement, tendant à faire revenir les enfants à un stade de traitement antérieur? Nos résultats semblent indiquer que oui. Dans l'acquisition du bilinguisme et l'acquisition précoce d'une langue étrangère, la comparaison entre ce que l'enfant peut faire dans sa langue première, ou sa langue forte, et ce qu'il fait dans la langue la plus faible est un aspect crucial de la recherche, qui nous place au carrefour des facteurs en jeu : le facteur typologique des spécificités des langues a et b (ou 1 et 2) avec l'appui mutuel qu'elles peuvent se prêter, ou au contraire le nécessaire apprentissage à entrer dans leurs mondes sémiotiques différents et à exploiter leurs ressources divergentes; le facteur du développement cognitif, l'enfant devenant de plus en plus capable d'aborder une tâche de façon globale en hiérarchisant et en planifiant ; le facteur linguistique enfin, qui met en évidence l'évolution des connecteurs d'une (ou de plusieurs) forme(s) à fonction sémantique indifférenciée, additive (et, et pi, cf. Jisa 1987) chez les jeunes enfants vers un développement de marqueurs différenciés de liens temporels, causaux et de but permettant de hiérarchiser la représentation des événements, puis (vers 10 ans) de s'adapter aux registres situationnels en variant les types de réalisation de l'hypotaxe (Gayraud, Jisa \& Viguié 2001). 


\section{RÉFÉRENCES}

ALLAL, L. (1999): La compétence langagière chez des enfants issus de limmigration tunisienne en France : Construction du récit et référence temporelle en français et en arabe, DEA de Sciences du Langage, option 'Acquisition des langues', Université Paris X.

ALLAL, L. (2000): «Enfants de migrants: un trait d'union entre deux langues et deux cultures», communication aux Journées d'Etudes France, pays de contact de langues, Tours, novembre 2000.

ALLAL, L. (en cours) : Acquisition linguistique et développement de la capacité narrative en français et en arabe tunisien chez des enfants issus de limmigration, doctorat de Sciences du langage, Université Paris X.

ANANE, Ch. (1998): Structure des récits en langue maternelle et en langue française chez des enfants tunisiens appartenant à deux environnements d'apprentissage. Mémoire de DEA de Sciences du Langage, Université Paris X.

ANANE Ch. (2001) : «Variation et hétérogénéité de récits en français de jeunes élèves tunisiens aux premiers stades de l'acquisition de cette langue». TRANEL 34/35 'Le changement linguistique. Evolution, variation, hétérogénéité', 323-338.

ANANE, Ch. (en cours): Acquisition linguistique et développement de la capacité narrative en français et en arabe tunisien chez des enfants scolarisés en Tunisie, doctorat de Sciences du langage, Université Paris X.

BERMAN Ruth \& Dan SLOBIN (1994): Different ways of relating events in narrative : a cross-linguistic developmental study. Hillsdale, NJ, Lawrence Erlbaum.

BLANCHE-BENVENISTE, C., (1997) : Approches de la langue parlée en français, Gap, Paris, Ophrys.

BOURDIN B. \& M. FAYOL (1994) : «Is written language production really more difficult than oral language production? » International Journal of Psychology 29, 591-620.

BRAHIM, A. (1997) : « De quelques cas de grammaticalisation en arabe tunisien », Revue de Lexicologie, $\mathrm{N}^{\circ} 12-$ 13, 'Les fondements théoriques du lexique', Actes du IV Colloque International de lexicologie organisé par l'ATAL, 105-127.

FAYOL Michel (2000) : «Comprendre et produire des textes écrits : l'exemple du récit ». In Kail M. \& M. Fayol (eds) L'acquisition du langage. Le langage en développement au-delà de trois ans. Paris: PUF, 183-213.

FAVARD M. \& J-M. PASSERAULT (1995) : «Évolution du rôle fonctionnel des connecteurs et de la planification écrite du récit chez les enfants de 7 à 11 ans ». Revue de Phonétique Appliquée 115-116-117, 'Psychologie et didactique', 198-212.

GAYRAUD, F., JISA H., VIGUIÉ, A. (2001) : «Utilisation des outils cohésifs comme indice de sensibilité au registre : une étude développementale ». AILE 14, 3-24.

HAIMAN John \& S.A. THOMPSON eds. (1989) : Clause-combining in grammar and discourse. Amsterdam, Benjamins.

JISA Harriet (1987): «Sentence connectors in French children's monologue performance ». Journal of Pragmatics 11, 607-621.

KLEIN W. \& C. von STUTTERHEIM (1989) : « Referential movement in descriptive and narrative discourse ». In R. Dietrich \& C.F. Graumann (Eds.) Language processing in social context. Amsterdam: Elsevier Science Publishers B.V. 
KOCH, P. (1995) : «Subordination, intégration syntaxique et "oralité” ». In H.L. Andersen \& G. Skytte éds., 'La subordination dans les langues romanes', Etudes Romanes 34, Copenhague : Museum Tusculanum Press, pp. 13-42.

LEVELT W. (1989) : Speaking. From intention to articulation. Cambridge, MA / London : Bradford Books MIT Press.

LORENZO ROSSELLO, C. de (2001), «Relations temporo-aspectuelles entre les procès dans le récit oral, en français et en castillan» In Macchi, Y., (Ed). Panorama de la linguistique hispanique Lille 2000. Collection 'UL3 Travaux de recherches', Presses de l'Université Lille III, 251-268.

MAYER, M. (1969) : Frog, where are you?, New York, Dial.

NOYAU, C. (1991) : Le temps dans le discours. Construction du récit, construction de la langue. Thèse d'habilitation. Université de Paris VIII. Vol.1.

NOYAU C., ed. (1998) : « Récit et temporalité dans l'acquisition bilingue du langage ». LINX 38.

NOYAU C., PAPROCKA, U. (2000) : «La représentation de structures événementielles par les apprenants: granularité et condensation ». Roczniki Humanistyczne, t.XLVIII, z. 5, Lublin, 2000 [Annales de Lettres et Sciences Humaines, vol.XLVIII, cahier 5, Lublin, Pologne], 87-121.

NOYAU C. \& DE LORENZO C., KIHLSTEDT M., PAPROCKA U., SANZ G., SCHNEIDER R. (sous presse) : « Two dimensions of the representation of complex event structures : granularity and condensation. Towards a typology of textual production ». In $\mathrm{H}$. Hendriks (ed.), The structure of learner language. Berlin : De Gruyter.

SANZ, G. (1999) «La proposición : una unidad semántico-conceptual para el estudio de la referencia en el discurso y de las relaciones interproposicionales ». In Cien años de investigación semántica de Michel Bréal a la actualidad. Actas del Congreso Internacional de Semántica. Universidad de La Laguna, octobre 1997, Madrid, Ediciones Clásicas, p. 905-916 (vol 2).

SCHLYTER Suzanne (1995): «Formes verbales du passé dans des interactions en langue forte et en langue faible ». AILE 6, 129-152.

SENEMAUD, M., (1997) : L'enfant et le récit : étude sur la temporalité dans les récits d'enfants en vue d'une recherche sur les enfants dysphasiques. Mémoire de Maîtrise de Sciences du Langage, Université Paris X.

SÉNÉMAUD, M., (1998) : L'enfant et le récit : étude sur la spatialité dans les récits d'enfants en vue d'une recherche sur les enfants dysphasiques, DEA de Sciences du Langage, option 'Acquisition des langues', Université Paris X.

SÉNÉMAUD, M. \& CHOI, J-N. (2000) : Variables temporelles dans des récits d'enfants français et d'enfants bilingues franco-coréens, Communication affichée au Colloque des Jeunes Linguistes, Paris, AFLA.

SÉNÉMAUD, M. (2001) : Comparaison de groupes d'enfants atteints / non atteints de troubles du langage, Communication affichée à la Journée « Jeunes Chercheurs en Linguistique Appliquée », Paris, AFLA.

SÉNÉMAUD, M. (en cours) : Capacité de production textuelle des enfants à retard de langage : analyse et remédiation, doctorat de Sciences du Langage, Université Paris X. 
